\title{
Design of Video Surveillance System based on Power Line Carrier and RFID
}

\author{
Xiaorui YUE, Jihua CHEN \\ Hubei Urban Construction Vocational And Technological College, Wuhan, China
}

Keywords: Power Line Carrier, Video Monitoring, Street Lamp Control, RFID.

\begin{abstract}
The idea of designing street lamp control system with MI200E power line carrier chip has been introduced. It puts emphasis on elaborating the interface of controller module and power line carrier module, hardware circuit design as well as the system software design. Testing results have shown that this system has realized good operation and management for street lamp control and its performance is stable and reliable.
\end{abstract}

\section{Introduction}

Power line communication technology is a communication method that adopts power line to transfer data and speech signal. This technology loads high frequency signal that carries signal to power line, adopts power line to make data transmission, modulate and demodulate through special power line, separates high-frequency signal from power line and transfers to terminal equipment [1]. On the basis of extensive grid distribution in China, this paper has studied and designed a control system for street lamp which adopts power line carrier transmission method.

\section{System Design}

Due to the big signal attenuation of power line during trans-transformer transmission, it can adopt GPRS wireless network communication to transfer based on practical demand or realize trans-regional data communication through accessing WAN through route. Managers only need to operate computer. Through making data transmission by power line, it can control the on/off status of street lamp and check the operation status of street lamp, so that it can realize timely and effective management control for street lamp.

\section{Design ideas}

Street lamp control system is composed of master control center, street lamp intelligent control center and street lamp control box. Distribution transformer has barrier effect for power carrier signal, so power carrier signal can only be transmitted within one distribution transformer region. Master control center can realize data transmission through GPRS wireless communication network or route and street lamp intelligent control center. After intelligent control center receiving order from master control center, transfer the order of monitor center to the street lamp control box of each branch through power line carrier. At the same time, street lamp intelligent control center detects the temperature, brightness, voltage, current and other situations of each lamp through power line carrier module, sends out abnormal voltage and current alarms, street lamp failure alarms, super high temperature alarm and other information to master control center to manage and control each street lamp.

\section{Hardware design}

It mainly designs the interface of controller module of street lamp control system and power line transmission module as well as power line transmission module.

1) MI200E power line carrier chip

The power transmission module adopts MI200E power line carrier communication chip, produced by Shanghai Miartech Company. It adopts complicated quadrature modulation principle, which is with great superiority in the power line transmission with fierce signal attenuation variation. Compared with current narrow band communication mode, spread spectrum 
communication mode and orthogonal frequency multiplexing technology, it can stop negative influence brought by the relation between phase and quadrature effectively. MI200E is power line carrier communication chip with high integration degree and high performance that makes optimization design for low voltage power line specially. It is with features of reliable communication and strong anti-interference ability etc, so that the user can embed the module into system easily [2].

2) Controller module

Select the 32-bit ARM processor of LM3S6916 as the controller of intelligent control center. It supports ARM Cortex-M3 core with the maximum frequency of $50 \mathrm{MHz}$. Integrated nested vectors interrupt controlling. Its greatest advantage lies in integrating $100 \mathrm{MHz}$ Ethernet when compared with other controllers [3]. When intelligent control center and master controller are in different LANs, computer connects with WAN through router. It can realize communication as long as making configuration for IP address or it can also adopt GPRS wireless network module to make data communication. Controller and power line transmission module adopt SPI interface. It does not need addressing operation and it is with full duplex communication. It is easy and with high efficiency and the highest speed can reach several Mb/s.

The CPU frequency of controller LM3S6916 adopts $6 \mathrm{MHz} 、 3.3 \mathrm{~V}$ for power supply. $25 \mathrm{MHz}$ crystal oscillator is used for the transmission of network data and the system adopts button reset operation. CS is the chip selection input of MI200E, SDO is series data output, SDI is series data input, SCK is series clock input. During order reading, set chip selection signal CS as low frequency. At this moment, SDO is high impedance state, series data is input by SDI, the rising edge of clock signal SCK is locked. During instruction writing, the trailing edge of data at clock signal SCK is output by SDO. After PLC-AC is connected with power, the data sending and receiving can be realized through the transmission way of power line carrier.

3) Power transmission module

Controller of intelligent control center realizes data transmission through power carrier module and sub-control box power carrier module. Due to the highly integrated features of MI200E power line carrier chip, it makes its peripheral circuit design very simple, so this design adopts MI200E as power line carrier communication chip.

The simulation power AVDD and digital power DVDD of MI200E are connected to $10 \mu \mathrm{F}$ electrolytic capacitor and $10 \mu \mathrm{F}$ electrolytic capacitor respectively and make filtering for the power. In circuit design, access magnetic beads between digital power DVDD and simulation power AVDD, this can decrease the interference of digital signal for simulation signal. To decrease the attack of $220 \mathrm{~V}$ power for power carrier chip, this design connects $5.1 \mathrm{M} \Omega$ and $220 \mathrm{k} \Omega$ resistances in series to power line on VAC+ and VAC- respectively. MI200E can select different carrier rates based on different requirements. This design adopts 1920b/s transmission speed and $12 \mathrm{MHz}$ crystal frequency. It outputs by $\mathrm{PA}$ and $\mathrm{PB}$ at $76.8 \mathrm{kHz}$ carrier signal. Carrier signal will be delivered to power line after coupling circuit. RAI+ and RAI- receive $76.8 \mathrm{kHz}$ carrier signal on power line. MI200E carrier chip makes corresponding data processing for data signal after demodulation.

\section{Software Design}

Software design adopts Keil Uvision 3 as programming tool of LM3S6916. Interrupt main program setting and check internal register of MI200E in every 2ms. During data sending, MI200E transfers frame header, baud rate and data length first at the speed of 200b/s; and then the user can reset mode register based on requirements and change the baud rate of send data. MI200E is with hardware automation checkout function and can read the comparison value from register directly. During data receiving, firstly write the baud rate and data length during data sending into register and check if the received data is correct or not after hardware accomplishing CRC test. The system is in data receiving status by default. 


\section{System Test}

Connect system control center module with intelligent control center module through internet; connect intelligent control center module with sub-control box module through power line. Many tests have been made for intelligent control center and sub-control box through network debugging assistant and power line carrier communication and realize reliable communication between them. Master control center realizes forced turn on and turn off of street lamp, upload system time, upload street lamp operation parameter through power line carrier and realize the monitoring and control for street lamp. The local port number is 4374 by default and the local IP address is 192.168.1.55. Set the port number of server that is intelligent control center as 5000 and IP address as 192.168.1.191. Many tests have been made for intelligent control center and sub-control box through network debugging assistant and power line carrier communication. Realize the mutual communication between them.

Testing results of Ethernet frame transfer protocol have shown that upload forced turn on or turn off order, street lamp sub-control box to return data SGGOPL<1KPGO00000000, which indicates the successful turn on or turn off of No.1 street lamp. Upload system time order to return data 0001UTGO, the corresponding sixteen hexadecimal data is 30303031555447302010120204 021646 OD OA, the system time is 20101202040216 46, which indicates time 2:16:46 on Thursday Dec. $2^{\text {nd }} 2010$. Upload street lamp operation parameter to return data 0002UPGP, the corresponding sixteen hexadecimal data is 3030303255504730 F1 4646000000000101 DE OD OA, the street lamp operation parameter is F1 $4646000000000101 \mathrm{DE}$, changed into decimal number as 2417070000011 222, which indicates relay switch status 241, street lamp operation mode 70 , street lamp forced/automatic mode 70 , street lamp brightness 0 , environment brightness 00, environment temperature 01 and CPU temperature 1222.

Power line is an extensive network. With this advantage, it does not need to set up network for street lamp system again and only needs to make use of existing distribution grid to make data transmission, which has decreased the cost of infrastructure construction and maintenance to a great extent. This paper selects MI200E as power line carrier communication module, which can realize stable and reliable data transmission. A system for street lamp control based on power line carrier has been studied and designed on this basis. The system realization has proven the feasibility of program design as well as the stable and reliable performance, which can provide reference for following "low carbon" economy.

\section{References}

[1] Cui Liu, Yanhu Li, Yingyue Zhang, Chuluo Yang, Hongbin Wu, Jingui Qin, and Yong Cao, Solution-Processed, Undoped, Deep-Blue Organic Light-Emitting Diodes Based on Starburst Oligofluorenes with a Planar Triphenylamine Core, Chemistry - A European Journal, 2012, 18(22), 6928-6934.

[2] Dalia Abdelhamid, Hulya Arslan, Yingyue Zhang, and Kathryn E. Uhrich, Role of Branching of Hydrophilic Domain on Physicochemical Properties of Amphiphilic Macromolecules, Polymer Chemistry, 2014, 5(4), 1457-1462.

[3] Dalia S. Abdelhamid, Yingyue Zhang, Daniel R. Lewis, Prabhas V. Moghe, William J. Welsh, and Kathryn E. Uhrich, Tartaric Acid-based Amphiphilic Macromolecules with Ether Linkages Exhibit Enhanced Repression of Oxidized Low Density Lipoprotein Uptake, Biomaterials, 2015, 53, 32-39.

[4] Yingyue Zhang, Jennifer W. Chan, Alysha Moretti, and Kathryn E. Uhrich, Designing Polymers with Sugar-based Advantages for Bioactive Delivery Applications, Journal of Controlled Release, 2015, 219, 355-368. 
[5] Yingyue Zhang, Qi Li, William J. Welsh, Prabhas V. Moghe, and Kathryn E. Uhrich, Micellar and Structural Stability of Nanoscale Amphiphilic Polymers: Implications for Anti-atherosclerotic Bioactivity, Biomaterials, 2016, 84, 230-240. 